\title{
Solution of Radiative Transfer In Anisotropic Plane-Parallel Atmosphere
}

\author{
Tasuku Tanaka ${ }^{1}$ \\ Earth Observation Research Center, National Space Development Agency of \\ Japan, 1-8-10 Harumi, Chuou-ku, Tokyo, 104-6023, Japan \\ E-mail:tanaka@eorc.nasda.go.jp \\ Menghua Wang \\ University of Maryland Baltimore County, Code970.2, NASA Goddard Space \\ Center, Greenbelt, MD20771 E-mail:wang@simbios.gsfc.nasa.gov \\ Tel:301-286-6421
}

\begin{abstract}
We solve the radiative transfer problem analytically in the anisotropic, plane-parallel atmosphere. Chandrasekhar formalized the radiative transfer process as a simultaneous, two-variable, non-linear, integral equation and obtained the analytical solution of the second approximation for the isotropic plane-parallel atmosphere. We obtain the second approximation for the anisotropic atmosphere, by integrating the first approximation multiplied by weighting functions which are products of the scattering phase functions. We truncate the second approximation and obtain the radiance at the top of the atmosphere as a quadratic equation with a logarithmic term in the optical thickness. We evaluate the second approximation of the radiance at the top of the atmosphere for Rayleigh scattering and the maritime aerosol atmosphere and compare them with both the exact solution and the single scattering approximation.
\end{abstract}

\section{Introduction}

In the area of satellite remote sensing, we observe the radiance at the top of the atmosphere (TOA) by instruments aboard satellites. And we retrieve the

1 Temporary address: c/o SIMBIOS Project, code 970.2, Goddard Space Flight Center, NASA, Greenbelt, Md. 20771, USA. E-mail:tanaka@simbios.gsfc.nasa.gov Tel:301-286-5624

Preprint submitted to Elsevier Science 7 March 2008 

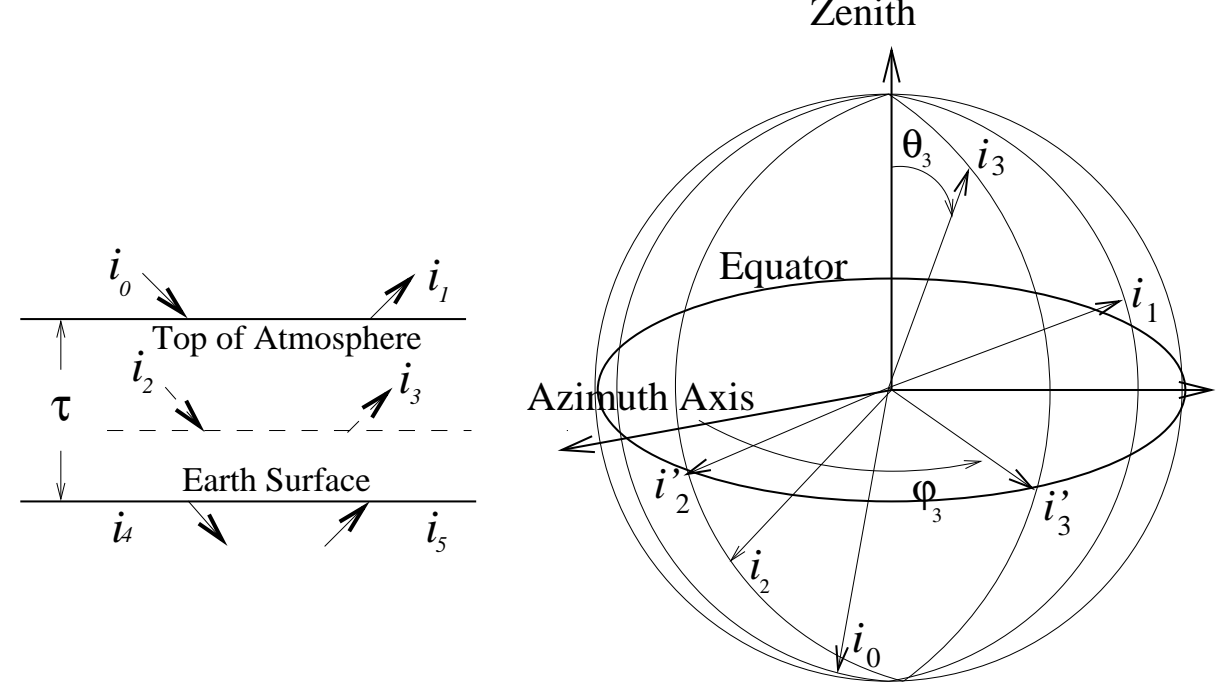

Fig. 1. Geometry of Radiative Transfer

surface reflectance and the optical thickness from the observed radiance (inversion problem). Since there are two unknown variables for one observation, there must be another observation or a relationship among them. In this paper we ignore the surface reflectance and seek the solution only for the optical thickness. The retrieval of the surface reflectance will be discussed in other occasions.

We show the geometry of the radiative transfer process in Fig. 1. The layer has the vertical optical thickness $\tau$. The direction of the input solar radiance $\vec{i}_{0}$ is the lower bound, the direction of observation by satellites $\vec{i}_{1}$ is the upper bound, the direction of the transmitted radiation to the Earth surface $\vec{i}_{4}$ is the lower bound, the intermediate direction $\vec{i}_{2}$ is the lower bound, and the other intermediate direction $\vec{i}_{3}$ is the upper bound. We employ the polar coordinate system in which the zenith angle $\theta$ is measured from the zenith and the azimuth angle $\varphi$ is measured from the projection of the solar direction onto the equator. We denote the cosine of the zenith angle and the azimuth angle of the direction $\vec{i}_{n}$ as $\mu_{n}$ and $\varphi_{n}$ respectively.

We usually employ the generalized reflectance $\rho$ in stead of radiance $I$,

$$
\rho\left(\tau, \vec{i}_{1}, \vec{i}_{0}\right)=\frac{\pi I}{F_{0}\left|\mu_{0}\right|},
$$

where $F_{0}$ is the solar irradiance. Since $\tau$ is considered sufficiently small to 1 , we have the first approximation,

$$
\rho=\frac{\tau P\left(i_{1}, i_{0}\right)}{4\left|\mu_{0}\right| \mu_{1}},
$$


where $P\left(\vec{i}_{1}, \vec{i}_{0}\right)$ is the scattering phase function. The first approximation, or the single scattering approximation has been applied to many analyses. Since the advent of Earth observing satellites, in particular the retrieval of the ocean chlorophyll concentration from Coastal Zone Color Scanner (CZCS), we need a more accurate formula. Many varieties of calculating methods have been proposed $[1,2]$. Most of them comprise the forward calculation and the lookup table. They do not give us the explicit form of $\rho$ by $\tau$. In this paper we seek the quadratic form of $\rho$ in $\tau$ as an explicit form of $\rho$ by $\tau$,

$$
\rho=c_{1} \tau+c_{2} \tau^{2}
$$

We employ the iterative integration of Chandrasekhar's integral equation to solve the inversion problem of the radiative transfer. The radiative transfer process in the atmosphere is governed by the integral equation, which was first introduced by Chandrasekhar[3]. He also obtained the explicit solution of radiative transfer in the second approximation for the isotropic plane-parallel atmosphere. We have obtained the explicit solution of the third approximation in the isotropic plane-parallel atmosphere [7]. In this paper we extend the second approximation developed by Chandrasekhar to the anisotropic atmosphere.

In Chandrasekhar's integral equation, there are two unknown functions: the scattering function, $S\left(\tau, \vec{i}_{1}, \vec{i}_{0}\right)$ and the transmitted function, $T\left(\tau, \vec{i}_{4}, \vec{i}_{0}\right)$, for which we seek the solutions (section two). We solve this simultaneous integral equation by successive iteration. The first approximation corresponds to the single scattering approximation. Substituting the first approximation into the integration in the original equations, we obtain the second iteration. Adding the first approximation to the second iteration, we obtain the second approximation for $S_{2}\left(\tau, \vec{i}_{1}, \vec{i}_{0}\right)$ and $T_{2}\left(\tau, \vec{i}_{4}, \vec{i}_{0}\right)$. We can continue this iterating process and obtain series $S_{n}\left(\tau, \overrightarrow{i_{1}}, \vec{i}_{0}\right)$ and $T_{n}\left(\tau, \vec{i}_{4}, \vec{i}_{0}\right)$. If the series $S_{n}\left(\tau, \vec{i}_{1}, \vec{i}_{0}\right)$ and $T_{n}\left(\tau, \vec{i}_{4}, \vec{i}_{0}\right)$ converge as $n$ approaches to $\infty$, it satisfies Chandrasekhar's integral equation (subsection 3.1). We decompose the second iteration into a power series expansion in $(-\tau)$ and obtain the quadratic form of the scattering function (subsection 3.2).

In the process to evaluate $S_{2}\left(\tau, \vec{i}_{1}, \vec{i}_{0}\right)$, we often encounter a sort of integration,

$$
U_{0}\left(\tau, \vec{i}_{0}\right)=\int_{0}^{1} \frac{\mu_{3}\left|\mu_{0}\right|}{\mu_{3}+\left|\mu_{0}\right|}\left(1-e^{-\frac{\tau}{\mu_{3}}-\frac{\tau}{\left|\mu_{0}\right|}}\right) \frac{d \mu_{3}}{\mu_{3}} .
$$

Expanding the term in the integral above into a power series in $(-\tau)$ and integrating indefinitelythose, we obtain 


$$
\begin{aligned}
U_{0}\left(\tau, \vec{i}_{0}\right) & =\int_{0}^{1}\left[-(-\tau)-\left(\frac{1}{\mu_{3}}+\frac{1}{\left|\mu_{0}\right|}\right) \frac{(-\tau)^{2}}{2 !}-\cdots\right] \frac{d \mu_{3}}{\mu_{3}} \\
& =\left[-\log \mu_{3}(-\tau)-\left(-\frac{1}{\mu_{3}}+\frac{\log \mu_{3}}{\left|\mu_{0}\right|}\right) \frac{(-\tau)^{2}}{2 !}-\frac{1}{\mu_{3}}-\cdots\right] .
\end{aligned}
$$

Substituting $\mu_{3}=1$ a t the upper integral limit, we can obtain the upper integral value. And the coefficients of nth power in $\tau$ are separately integrated. However at the lower limit, each integrated term becomes $\infty$, or has singularities at $\mu_{3}=0$. For the lower limit, we must sum up all the terms on the interval $(\epsilon, 1)$ and then make $\epsilon$ approach to 0 . Briefly we can not change the order of "lim" and " $\sum$ " at the lower integral limit.(subsection 3.3)

The problem of the radiative transfer is, thus, deduced to evaluate the value of the series expansion at the singularity point. The authors evaluated values of several series expansions at the singularity point, which are necessary to obtain the second and third approximation for the isotropic atmosphere [7].

We show that, for the anisotropic atmosphere, we can exchange the order of "lim" and " $\sum$ " for the higher powers of $\tau$. If we can exchange the order, we can integrate each power in $(-\tau)$ separately (subsection 3.3). Based on this consideration, we show that the coefficients of the quadratic form of the second iteration expressed as the surface integrations of products of the scattering phase functions on the half unit sphere (subsection 3.4 and 3.5).

Finally in section four, we evaluate the second approximations numerically for the Rayleigh scattering and the maritime aerosol atmosphere. And the results are compared with the single scattering approximation and the exact solutions.

\section{Radiation Transfer Process}

The scattered radiance at the top of the atmosphere (TOA), $I\left(0, \vec{i}_{1}\right)$, is expressed with the incident intensity $I\left(0, i_{0}\right)$ and the scattering function $S\left(\tau, i_{1}, i_{0}\right)$ as

$$
I\left(0, \vec{i}_{1}\right)=\frac{1}{4 \pi \mu_{1}} \int_{L} S\left(\tau, \vec{i}_{1}, \vec{i}_{0}\right) I\left(0, \vec{i}_{0}\right) d \Omega_{0},
$$

where $\Omega_{0}$ is the solid angle subtended around the input direction $\vec{i}_{0}$ and integral domain is the lower half of the unit sphere. In the same manner the intensity 
transmitted to the Earth surface is expressed as

$$
I\left(0, \vec{i}_{4}\right)=\frac{1}{4 \pi\left|\mu_{4}\right|} \int_{L} T\left(\tau, \vec{i}_{4}, \vec{i}_{0}\right) I\left(0, \vec{i}_{0}\right) d \Omega_{0}+\exp \left(-\frac{\tau}{\left|\mu_{4}\right|}\right) I\left(0, \vec{i}_{4}\right) .
$$

Chandrasekhar's integral equation is expressed [3],

$$
\begin{aligned}
& \left(\frac{1}{\mu_{1}}+\frac{1}{\left|\mu_{0}\right|}\right) S\left(\tau, \vec{i}_{1}, \vec{i}_{0}\right)=\left[1-\exp \left(\frac{(-\tau)}{\mu_{1}}+\frac{(-\tau)}{\left|\mu_{0}\right|}\right)\right] P\left(\vec{i}_{1}, \vec{i}_{0}\right) \\
& +\int_{U} P\left(\vec{i}_{1}, \vec{i}_{3}\right) S\left(\tau, \vec{i}_{3}, \vec{i}_{0}\right) \frac{d \Omega_{3}}{4 \pi \mu_{3}}-\exp \left(-\frac{\tau}{\left|\mu_{0}\right|}\right) \int_{U} T\left(\tau, \vec{i}_{1}, \vec{i}_{3}\right) P\left(\vec{i}_{3}, \vec{i}_{0}\right) \frac{d \Omega_{3}}{4 \pi \mu_{3}} \\
& +\int_{L} S\left(\tau, \vec{i}_{1}, \vec{i}_{2}\right) P\left(\vec{i}_{2}, \vec{i}_{0}\right) \frac{d \Omega_{2}}{4 \pi\left|\mu_{2}\right|}-\exp \left(-\frac{\tau}{\mu_{1}}\right) \int_{L} P\left(\vec{i}_{1}, \vec{i}_{2}\right) T\left(\tau, \vec{i}_{2}, \vec{i}_{0}\right) \frac{d \Omega_{2}}{4 \pi\left|\mu_{2}\right|} \\
& +\int_{U} \int_{L} S\left(\tau, \vec{i}_{1}, \vec{i}_{2}\right) P\left(\vec{i}_{2}, \vec{i}_{3}\right) S\left(\tau, \vec{i}_{3}, \vec{i}_{0}\right) \frac{d \Omega_{2}}{4 \pi\left|\mu_{2}\right|} \frac{d \Omega_{3}}{4 \pi \mu_{3}} \\
& -\int_{U} \int_{L} T\left(\tau, \overrightarrow{i_{1}}, \overrightarrow{i_{3}}\right) P\left(\vec{i}_{3}, \vec{i}_{2}\right) T\left(\tau, \overrightarrow{i_{2}}, \overrightarrow{i_{0}}\right) \frac{d \Omega_{2}}{4 \pi\left|\mu_{2}\right|} \frac{d \Omega_{3}}{4 \pi \mu_{3}}, \\
& \left(\frac{1}{\left|\mu_{4}\right|}-\frac{1}{\left|\mu_{0}\right|}\right) T\left(\tau, \vec{i}_{4}, \vec{i}_{0}\right)=\left[\exp \left(-\frac{\tau}{\left|\mu_{0}\right|}\right)-\exp \left(-\frac{\tau}{\left|\mu_{4}\right|}\right)\right] P\left(\vec{i}_{4}, \vec{i}_{0}\right) \\
& +\int_{L} P\left(\vec{i}_{4}, \vec{i}_{2}\right) T\left(\tau, \vec{i}_{2}, \vec{i}_{0}\right) \frac{d \Omega_{2}}{4 \pi\left|\mu_{2}\right|}-\exp \left(-\frac{\tau}{\left|\mu_{4}\right|}\right) \int_{U} P\left(\vec{i}_{4}, \vec{i}_{3}\right) S\left(\tau, \vec{i}_{3}, \vec{i}_{0}\right) \frac{d \Omega_{3}}{4 \pi \mu_{3}} \\
& +\exp \left(-\frac{\tau}{\left|\mu_{0}\right|}\right) \int_{U} S\left(\tau, \vec{i}_{4}, \vec{i}_{3}\right) P\left(\vec{i}_{3}, \vec{i}_{0}\right) \frac{d \Omega_{3}}{4 \pi \mu_{3}}-\int_{L} T\left(\tau, \vec{i}_{4}, \vec{i}_{2}\right) P\left(\vec{i}_{2}, \vec{i}_{0}\right) \frac{d \Omega_{2}}{4 \pi\left|\mu_{2}\right|} \\
& +\int_{U} \int_{L} S\left(\tau, \vec{i}_{4}, \vec{i}_{3}\right) P\left(\vec{i}_{3}, \vec{i}_{2}\right) T\left(\tau, \vec{i}_{2}, \vec{i}_{0}\right) \frac{d \Omega_{2}}{4 \pi\left|\mu_{2}\right|} \frac{d \Omega_{3}}{4 \pi \mu_{3}} \\
& -\int_{U} \int_{U} T\left(\tau, \vec{i}_{4}, \vec{i}_{2}\right) P\left(\vec{i}_{2}, \vec{i}_{3}\right) S\left(\tau, \vec{i}_{3}, \vec{i}_{0}\right) \frac{d \Omega_{2}}{4 \pi\left|\mu_{2}\right|} \frac{d \Omega_{3}}{4 \pi \mu_{3}} .
\end{aligned}
$$

The cosine of the zenith angle in the lower bound directions, which has a "even index", is negative. For the convenience of the following evaluation, we replace $\mu_{n}$ by a new parameter, $\mu_{n}^{-}$which is equal to $-\mu_{n}$ for $n=$ even. Using the new variables, the integration on the lower half sphere is changed to the integration on the upper half unite sphere, shown below

$$
\int_{L} \frac{f\left(\mu_{2}, \cdot \cdot\right) d \Omega_{2}}{4 \pi\left|\mu_{2}\right|}=\int_{0}^{2 \pi} d \varphi_{2} \int_{0}^{-1} \frac{f\left(\mu_{2}, \cdot, \cdot\right)\left(-d \mu_{2}\right)}{4 \pi\left(-\mu_{2}\right)}
$$




$$
=\int_{0}^{2 \pi} d \varphi_{2} \int_{0}^{1} \frac{f\left(-\mu_{2}^{-}, \cdot, \cdot\right) d \mu_{2}^{-}}{4 \pi \mu_{2}^{-}}=\int_{U} f\left(-\mu_{2}^{-}, \cdot, \cdot\right) \frac{d \Omega_{2}^{-}}{4 \pi \mu_{2}^{-}} .
$$

Since we adopt the new variable $\mu_{2}^{-}$and the integral domain is changed to the upper half sphere, we ommite the $U$ for the integral domain unless the domain has a special meaning. The cosine of the angle between the upper and lower bound directions is thus expressed as below

$$
\begin{array}{r}
\cos \left(\theta_{e, o}\right)=\mu_{e} \mu_{o}+\left(1-\mu_{e}^{2}\right)^{1 / 2}\left(1-\mu_{o}^{2}\right)^{1 / 2} \cos \left(\varphi_{e}-\varphi_{o}\right) \\
=-\mu_{e}^{-} \mu_{o}+\left(1-\left(\mu_{e}^{-}\right)^{2}\right)^{1 / 2}\left(1-\mu_{o}^{2}\right)^{1 / 2} \cos \left(\varphi_{e}-\varphi_{o}\right) .
\end{array}
$$

The phase function is assumed as a function of the angle $\theta_{m n}$ between the directions $m$ and $n$ and is normalized in the whole solid angle $4 \pi$. We express the phase function $P\left(i_{m}, i_{n}\right)$ as below,

$$
P\left(i_{m}, i_{n}\right)=P\left(\cos \theta_{m n}\right)=\sum_{j=0}^{\infty} w_{j} P_{j}\left(\cos \theta_{m n}\right)
$$

where $P_{j}(x)$ is the Legendre function of the first kind.

The input from the upper surface is the solar irradiance, $F_{0}$, and is given with the Dirac delta function

$$
I(0, \vec{i})=F_{0} \delta\left(\vec{i}-\vec{i}_{0}\right) .
$$

Substituting the solar irradiance, we obtain the generalized reflectance from the upper layer

$$
\rho\left(0, \vec{i}_{1}\right)=\frac{S\left(\tau, \vec{i}_{1}, \vec{i}_{0}\right)}{4 \mu_{1}\left|\mu_{0}\right|} .
$$

\section{Second Approximation}

\subsection{Azimuth Integration and Decomposition of Second Iteration}

The simultaneous integral equations of radiative transfer can be solved by successive iteration. The first iterations are the first terms on the right-hand side in equations (8) and (9), expressed below 


$$
\begin{gathered}
S_{1}\left(\tau, \vec{i}_{1}, \vec{i}_{0}\right)=\left(\frac{1}{\mu_{1}}+\frac{1}{\mu_{0}^{-}}\right)^{-1}\left[1-\exp \left(\frac{(-\tau)}{\mu_{1}}+\frac{(-\tau)}{\mu_{0}^{-}}\right)\right] P\left(\vec{i}_{1}, \vec{i}_{0}\right) \\
T_{1}\left(\tau, \vec{i}_{4}, \vec{i}_{0}\right)=\left(\frac{1}{\mu_{4}^{-}}-\frac{1}{\mu_{0}^{-}}\right)^{-1}\left(\exp \left(-\frac{\tau}{\mu_{0}^{-}}\right)-\exp \left(-\frac{\tau}{\mu_{4}^{-}}\right)\right) P\left(\vec{i}_{4}, \vec{i}_{0}\right)
\end{gathered}
$$

A new function $U_{1}\left(\tau, \mu_{0}, \mu_{1}\right)$ is defined as

$$
U_{1}\left(\tau, \mu_{1}, \mu_{0}^{-}\right)=\left(\frac{1}{\mu_{1}}+\frac{1}{\mu_{0}^{-}}\right)^{-1}\left[1-\exp \left(\frac{(-\tau)}{\mu_{1}}+\frac{(-\tau)}{\mu_{0}^{-}}\right)\right] .
$$

Using $U_{1}\left(\tau, \mu_{0}^{-}, \mu_{1}\right), T_{1}\left(\tau, \vec{i}_{4}, \vec{i}_{0}\right)$ is rewritten as

$$
T_{1}\left(\tau, \vec{i}_{4}, \vec{i}_{0}\right)=\exp \left(-\frac{\tau}{\mu_{0}^{-}}\right) U_{1}\left(\tau, \mu_{4}^{-},\left(-\mu_{0}^{-}\right)\right) P\left(\vec{i}_{4}, \vec{i}_{0}\right)
$$

Substituting the first iterations into the integrals in the original equations, the second iterations, $\Delta S_{2}\left(\tau, \vec{i}_{1}, \vec{i}_{0}\right)$ is expressed

$$
\begin{aligned}
& \Delta S_{2}\left(\tau, \vec{i}_{1}, \vec{i}_{0}\right)=\left(\frac{1}{\mu_{1}}+\frac{1}{\mu_{0}^{-}}\right)^{-1} \\
& {\left[\int P\left(\vec{i}_{1}, \vec{i}_{3}\right) P\left(\vec{i}_{3}, \vec{i}_{0}\right) U_{1}\left(\mu_{0}^{-}\right) \frac{d \Omega_{3}}{4 \pi \mu_{3}}-\int P\left(\vec{i}_{1}, \vec{i}_{3}\right) P\left(\vec{i}_{3}, \vec{i}_{0}\right) U_{1}\left(-\mu_{1}\right) \frac{d \Omega_{3}}{4 \pi \mu_{3}}\right.} \\
&+ \int P\left(\vec{i}_{1}, \vec{i}_{2}\right) P\left(\vec{i}_{2}, \vec{i}_{0}\right) U_{1}\left(\mu_{1}\right) \frac{d \Omega_{2}^{-}}{4 \pi \mu_{2}^{-}}-\int P\left(\vec{i}_{1}, \vec{i}_{2}\right) P\left(\vec{i}_{2}, \vec{i}_{0}\right) U_{1}\left(-\mu_{0}^{-}\right) \frac{d \Omega_{2}}{4 \pi \mu_{2}^{-}} \\
&-\left\{\exp \left(-\frac{\tau}{\mu_{0}^{-}}-\frac{\tau}{\mu_{1}}\right)-1\right\} \times \\
&\left\{\int P\left(\vec{i}_{1}, \vec{i}_{3}\right) P\left(\vec{i}_{3}, \vec{i}_{0}\right) U_{1}\left(-\mu_{1}\right) \frac{d \Omega_{3}}{4 \pi \mu_{3}}+\int P\left(\vec{i}_{1}, \vec{i}_{2}\right) P\left(\vec{i}_{2}, \vec{i}_{0}\right) U_{1}\left(-\mu_{0}^{-}\right) \frac{d \Omega_{2}^{-}}{4 \pi \mu_{2}^{-}}\right\} \\
&+\iint P\left(\overrightarrow{i_{1}}, \overrightarrow{i_{2}}\right) P\left(\overrightarrow{i_{2}}, \vec{i}_{3}\right) P\left(\overrightarrow{i_{3}}, \vec{i}_{0}\right) U_{1}\left(\mu_{1}, \mu_{2}^{-}\right) U_{1}\left(\mu_{3}, \mu_{0}^{-}\right) \frac{d \Omega_{2}^{-}}{4 \pi \mu_{2}^{-}} \frac{d \Omega_{3}}{4 \pi \mu_{3}} \\
&\left.\left.-\iint P\left(\vec{i}_{1}, \vec{i}_{3}\right) P\left(\vec{i}_{3}, \vec{i}_{2}\right) P\left(\vec{i}_{2}, \vec{i}_{0}\right) U_{1}\left(-\mu_{0}^{-}, \mu_{2}^{-}\right) U_{1}\left(-\mu_{1}, \mu_{3}\right)\right) \frac{d \Omega_{2}^{-}}{4 \pi \mu_{2}^{-}} \frac{d \Omega_{3}}{4 \pi \mu_{3}}\right] \\
&-\left\{\exp \left(-\frac{\tau}{\mu_{0}^{-}}-\frac{\tau}{\mu_{1}}\right)-1\right\} \\
&\left.\left.\times \iint P\left(\vec{i}_{1}, \overrightarrow{i_{3}}\right) P\left(\overrightarrow{i_{3}}, \vec{i}_{2}\right) P\left(\overrightarrow{i_{2}}, \vec{i}_{0}\right) U_{1}\left(-\mu_{0}^{-}, \mu_{2}^{-}\right) U_{1}\left(-\mu_{1}, \mu_{3}\right)\right) \frac{d \Omega_{2}^{-}}{4 \pi \mu_{2}^{-}} \frac{d \Omega_{3}}{4 \pi \mu_{3}}\right]
\end{aligned}
$$

where the arguments in the functions $U\left(\tau, \vec{i}_{1}, \vec{i}_{0}\right)$ are omitted except for the significant ones. 
Using the addition theorem of the Legendre function, we decompose the phase function into the the Bi-Legendre functions, $P_{l}^{m}\left(\mu_{3}\right)$, as

$$
\begin{gathered}
P\left(\vec{i}_{1}, \vec{i}_{3}\right)=\left\{\sum_{m=0}^{\infty}\left(2-\delta_{0, m}\right)\left(\sum_{l=m}^{\infty} w_{l}^{m} P_{l}^{m}\left(\mu_{1}\right) P_{l}^{m}\left(\mu_{3}\right)\right) \cos m\left(\varphi_{3}-\varphi_{1}\right)\right\}( \\
P\left(\vec{i}_{3}, \vec{i}_{0}\right)=\left\{\sum_{m=0}^{\infty}\left(2-\delta_{0, m}\right)\left(\sum_{k=m}^{\infty} w_{k}^{m} P_{k}^{m}\left(\mu_{3}\right) P_{k}^{m}\left(-\mu_{0}^{-}\right)\right) \cos m\left(\varphi_{3}-\varphi_{0}\right)\right\},
\end{gathered}
$$

where $\delta_{0, m}$ and $w_{l}^{m}$ are given as below,

$$
\begin{aligned}
& \delta_{0, m}=1 \quad(m=0), \quad \delta_{0, m}=0 \quad(m \neq 0), \\
& w_{l}^{m}=w_{l} \frac{(l-m) !}{(l+m) !}
\end{aligned}
$$

Due to the orthogonality of the functions $P_{l}^{m}\left(\mu_{3}\right) \cos m\left(\varphi_{3}-\varphi_{1}\right)$ for different $m$, we obtain the integration

$$
\begin{aligned}
& \frac{1}{2 \pi} \int_{0}^{2 \pi} P\left(\vec{i}_{1}, \vec{i}_{3}\right) P\left(\vec{i}_{3}, \vec{i}_{0}\right) d \varphi_{3} \\
= & \sum_{m=0}^{\infty}\left(2-\delta_{0, m}\right)\left(\sum_{l, k=m}^{\infty}(-1)^{k} w_{l}^{m} w_{k}^{m} P_{l}^{m}\left(\mu_{1}\right) P_{k}^{m}\left(\mu_{0}^{-}\right) P_{l}^{m}\left(\mu_{3}\right) P_{k}^{m}\left(\mu_{3}\right)\right) \\
\times & \cos m\left(\varphi_{1}-\varphi_{0}\right) .
\end{aligned}
$$

In the same manner, we obtain the integration of the triple product of phase functions

$$
\begin{aligned}
& \int_{0}^{2 \pi} \int_{0}^{2 \pi} P\left(\vec{i}_{1}, \vec{i}_{2}\right) P\left(\vec{i}_{2}, \vec{i}_{3}\right) P\left(\vec{i}_{3}, \vec{i}_{0}\right) \frac{d \varphi_{2}}{2 \pi} \frac{d \varphi_{3}}{2 \pi} \\
= & \int_{0}^{2 \pi} \int_{0}^{2 \pi}\left[\sum_{m=0}^{\infty}\left(2-\delta_{0, m}\right)\left\{\sum_{l=m}^{\infty} w_{l}^{m} P_{l}^{m}\left(\mu_{1}\right) P_{l}^{m}\left(-\mu_{2}^{-}\right)\right\} \cos m\left(\varphi_{2}-\varphi_{1}\right)\right] \\
\times & {\left[\sum_{m=0}^{\infty}\left(2-\delta_{0, m}\right)\left\{\sum_{l=m}^{\infty} w_{l}^{m} P_{l}^{m}\left(-\mu_{2}^{-}\right) P_{l}^{m}\left(\mu_{3}\right)\right\} \cos m\left(\varphi_{3}-\varphi_{2}\right)\right] \frac{d \varphi_{2}}{2 \pi} } \\
\times & {\left[\sum_{m=0}^{\infty}\left(2-\delta_{0, m}\right)\left\{\sum_{l=m}^{\infty} w_{l}^{m} P_{l}^{m}\left(\mu_{3}\right) P_{l}^{m}\left(-\mu_{0}^{-}\right)\right\} \cos m\left(\varphi_{0}-\varphi_{3}\right)\right] \frac{d \varphi_{3}}{2 \pi} } \\
= & \sum_{m=0}^{\infty}\left(2-\delta_{0, m}\right) \cos m\left(\varphi_{3}-\varphi_{1}\right)\left[\sum_{a, b, c=m}^{\infty}(-1)^{a+b+c}\{\right.
\end{aligned}
$$




$$
\left.\left.w_{a}^{m} w_{b}^{m} w_{c}^{m} P_{a}^{m}\left(\mu_{1}\right) P_{a}^{m}\left(\mu_{2}\right) P_{b}^{m}\left(\mu_{2}\right) P_{b}^{m}\left(\mu_{3}\right) P_{c}^{m}\left(\mu_{3}\right) P_{c}^{m}\left(\mu_{0}\right)\right\}\right]
$$

We define an integration, $Q_{l, k}^{m}\left(\tau, \mu_{0}^{-}\right)$, as

$$
Q_{l, k}^{m}\left(\tau, \mu_{0}^{-}\right)=\int_{0}^{1} P_{l}^{m}\left(\mu_{3}\right) P_{k}^{m}\left(\mu_{3}\right) U_{1}\left(\tau, \mu_{1}, \mu_{0}^{-}\right) \frac{d \mu_{3}}{\mu_{3}} .
$$

Using $Q_{l, k}^{m}\left(\tau, \mu_{0}^{-}\right)$, we obtain $\Delta S_{2}$

$$
\begin{aligned}
& \quad \Delta S_{2}\left(\tau, \vec{i}_{1}, \vec{i}_{0}\right)=\frac{1}{2}\left(\frac{1}{\mu_{1}}+\frac{1}{\mu_{0}^{-}}\right)^{-1} \sum_{m=0}^{\infty}\left(2-\delta_{0, m}\right) \cos m\left(\varphi_{1}-\varphi_{0}\right) \times \\
& {\left[\sum _ { l , k = m } ^ { \infty } w _ { l } ^ { m } w _ { k } ^ { m } \left\{P_{l}^{m}\left(\mu_{1}\right) P_{k}^{m}\left(\mu_{0}^{-}\right)(-1)^{k}\left\{Q_{l, k}^{m}\left(\tau, \mu_{0}^{-}\right)-(-1)^{l+k} Q_{l, k}^{m}\left(\tau,-\mu_{0}^{-}\right)\right\}\right.\right.} \\
& \quad+\sum_{l, k=m}^{\infty} w_{l}^{m} w_{k}^{m}\left\{P_{l}^{m}\left(\mu_{1}\right) P_{k}^{m}\left(\mu_{0}^{-}\right)(-1)^{l}\left\{Q_{l, k}^{m}\left(\tau, \mu_{1}\right)-(-1)^{l+k} Q_{l, k}^{m}\left(\tau,-\mu_{1}\right)\right\}\right. \\
& \quad-\left\{\exp \left(-\frac{\tau}{\mu_{1}}-\frac{\tau}{\mu_{0}^{-}}\right)-1\right\} \\
& \times \sum_{l, k=m}^{\infty} w_{l}^{m} w_{k}^{m}\left\{P_{l}^{m}\left(\mu_{1}\right) P_{k}^{m}\left(\mu_{0}^{-}\right)(-1)^{l}\left\{Q_{l, k}^{m}\left(-\mu_{0}^{-}\right)+(-1)^{l+k} Q_{l, k}^{m}\left(-\mu_{1}\right)\right\}\right. \\
& +\frac{1}{2} \sum_{a, b, c=m}^{\infty}(-1)^{a+b+c} w_{a}^{m} w_{b}^{m} w_{c}^{m} P_{a}^{m}\left(\mu_{1}\right) P_{c}^{m}\left(\mu_{0}^{-}\right) Q_{a, b}^{m}\left(\tau, \mu_{1}\right) Q_{b, c}^{m}\left(\tau, \mu_{0}^{-}\right) \\
& \left.-\frac{1}{2} \sum_{a, b, c=m}^{\infty}(-1)^{b} w_{a}^{m} w_{b}^{m} w_{c}^{m} P_{a}^{m}\left(\mu_{1}\right) P_{c}^{m}\left(\mu_{0}^{-}\right) Q_{a, b}^{m}\left(\tau,-\mu_{1}\right) Q_{b, c}^{m}\left(\tau,-\mu_{0}^{-}\right)\right] \\
& -\left\{\exp \left(-\frac{\tau}{\mu_{0}^{-}}-\frac{\tau}{\mu_{1}}\right)-1\right\} \\
& \left.\times \frac{1}{2} \sum_{a, b, c=m}^{\infty}(-1)^{b} w_{a}^{m} w_{b}^{m} w_{c}^{m} P_{a}^{m}\left(\mu_{1}\right) P_{c}^{m}\left(\mu_{0}^{-}\right) Q_{a, b}^{m}\left(\tau,-\mu_{1}\right) Q_{b, c}^{m}\left(\tau,-\mu_{0}^{-}\right)\right] . \quad(27)
\end{aligned}
$$

In the above equation, the sign of each term is determined by the choice of $l, k, a, b, c$. This determination originates from the even/odd characteristic of the Bi-Legendre functions.

\subsection{Zenith Integration of Second Iteration}

The second iteration $\Delta S_{2}\left(\tau, i_{1}, i_{0}\right)$ is expressed in equation (27) and its component, $Q_{l, k}^{m}(\tau, \mu)$, has power a series expansion in $\tau$. In order to obtain the quadratic form of $\Delta S_{2}\left(\tau, i_{1}, i_{0}\right)$, we decompose $Q_{l, k}^{m}\left(\tau, \mu_{0}^{-}\right)$into the power se- 
ries expansion in $(-\tau)$. First we integrate $U_{2}^{0}\left(\tau, \mu_{0}^{-}\right)$and is evaluated in the author's previous work [7] given below,

$$
\begin{aligned}
& U_{2}^{0}\left(\tau, i_{0}^{-}\right)=\int_{0}^{1} U_{1}\left(\tau, \mu, \mu_{0}^{-}\right) \frac{d \mu}{\mu}=\int_{0}^{1}\left(1-\exp \left(-\frac{\tau}{\mu}-\frac{\tau}{\mu_{0}^{-}}\right)\right) \frac{\mu_{0}^{-} d \mu}{\mu_{0}^{-}+\mu} \\
= & (-\tau)\left[(C+\operatorname{eax}(\tau)) \sum_{r=0}^{\infty} \frac{\left(-\tau / \mu_{0}\right)^{r}}{(r+1) r !}-\sum_{r=0}^{\infty}\left(\sum_{n=0}^{\infty} \frac{(-\tau)^{n}}{(r+1+n) n !}\right) \frac{\left(-\tau / \mu_{0}\right)^{r}}{(r+1) r !}\right],
\end{aligned}
$$

where the constant $C$ and the function $\operatorname{eax}(p)$ are given as

$$
\begin{aligned}
& C=\gamma+\log \tau=0.5772 \cdots+\log \tau \\
& \operatorname{eax}(p)=\sum_{n=1}^{\infty} \frac{(-1)^{n} p^{n}}{n n !}
\end{aligned}
$$

where $\gamma$ is the Euler constant.

The function $U_{2}^{0}\left(\tau, \mu_{0}\right)$ is truncated as

$$
U_{2}^{0}\left(\tau, \mu_{0}^{-}\right)=(C-1)(-\tau)+\left\{1+\left(C-\frac{1}{2}\right) \frac{1}{\mu_{0}^{-}}\right\} \frac{(-\tau)^{2}}{2 !}+\cdots
$$

We integrate the moment integration, $U_{2}^{n}\left(\tau, \mu_{0}^{-}\right)$, as below,

$$
U_{2}^{n}\left(\tau, \mu_{0}^{-}\right)=\int_{0}^{1} \mu_{3}^{n} U_{1}\left(\tau, \mu_{3}, \mu_{0}^{-}\right) \frac{d \mu_{3}}{\mu_{3}} .
$$

$U_{2}^{n}\left(\tau, \mu_{0}^{-}\right)$is evaluated by partial integration and recurrence relationships (refer to appendix 2). The coefficients of the series expansion of $U_{2}^{n}\left(\tau, \mu_{0}^{-}\right)$in the power $(-\tau)$ are also polynomials of $1 / \mu_{0}^{-}$. The truncated form of $U_{2}^{n}\left(\tau, \mu_{0}^{-}\right)$ for $n \geq 1$ is expressed below.

$$
U_{2}^{n}\left(\tau, \mu_{0}^{-}\right)=(-1)^{n}(-\tau)+u_{2,0}^{n} \frac{(-\tau)^{2}}{2 !}+u_{2,1}^{n} \frac{(-\tau)^{2}}{2 !} \frac{1}{\mu_{0}^{-}}+\cdots
$$

where $u_{l, k}^{n}$ is the coefficient of $\frac{(-\tau)^{n}}{n !\left(\mu_{0}^{-}\right)^{k}}$ and is given in the appendix 2. It is noted that the coefficient of the first power $(-\tau)$ does not include $\mu_{0}^{-}$. Substituting $U_{2}^{n}$ into equation $(26)$, we obtain $Q_{l, k}^{m}(\tau, \mu)$ as a power series expansion in $(-\tau)$ 


$$
\begin{aligned}
& Q_{l, k}^{m}\left(\tau, \mu_{0}\right)=\int_{0}^{1} \sum_{n=0}^{m_{l k}} a_{n}^{m l k} \mu_{3}^{n} U_{1}\left(\tau, \mu_{3}, \mu_{0}^{-}\right) \frac{d \mu_{3}}{\mu_{3}} \\
= & \left(\sum_{n=0}^{m_{l k}} a_{n}^{m l k} u_{1,0}^{n}\right)(-\tau)+\left(\sum_{n=0}^{m_{l k}} a_{n}^{m l k} u_{2,0}^{n}\right) \frac{(-\tau)^{2}}{2 !}+\left(\sum_{n=0}^{m_{l k}} a_{n}^{m l k} u_{2,1}^{n}\right) \frac{(-\tau)^{2}}{2 !} \frac{1}{\mu_{0}^{-}}+\cdots \\
= & q_{1,0}^{m l k}(-\tau)+q_{2,0}^{m l k} \frac{(-\tau)^{2}}{2 !}+q_{2,1}^{m l k} \frac{(-\tau)^{2}}{2 !} \frac{1}{\mu_{0}^{-}}+\cdots
\end{aligned}
$$

where $a_{n}^{m l k}$ is the coefficient of the $n$ degree power of the product of the BiLegendre functions $P_{l}^{m}\left(\mu_{3}\right)$ and $P_{k}^{m}\left(\mu_{3}\right)$. The coefficient $q_{d, e}^{m l k}$ is given as below,

$$
q_{d, e}^{m l k}=\sum_{n=0}^{m_{l} k} a_{n}^{m l k} u_{d, e}^{n} .
$$

It is noted that the coefficient of the first power $(-\tau)$ in $Q_{l, k}^{m}\left(\tau, \mu_{0}\right)$ does not include $\mu_{0}^{-}$.

Substituting the equation (34) into the first term in the bracket of equation (27), we obtain following.

$$
\begin{aligned}
& Q_{l, k}^{m}\left(\tau, \mu_{0}^{-}\right)-(-1)^{l+k} Q_{l, k}^{m}\left(\tau,-\mu_{0}^{-}\right)=q_{2,1}^{m l k} \frac{(-\tau)^{2}}{2 !} \frac{2}{\mu_{0}^{-}}+\cdots(l+k=\text { even }) \\
& =2 q_{1,0}^{m l k}(-\tau)+2 q_{2,0}^{m l k} \frac{(-\tau)^{2}}{2 !}+\cdots \quad(l+k=\text { odd })
\end{aligned}
$$

And for the case $(l+k=o d d)$, adding the second term to the above, we obtain following,

$$
\begin{aligned}
& P_{l}^{m}\left(\mu_{1}\right) P_{k}^{m}\left(\mu_{0}\right)(-1)^{k}\left\{Q_{l, k}^{m}\left(\mu_{0}\right)-(-1)^{l+k} Q_{l, k}^{m}\left(-\mu_{0}\right)\right\} \\
+ & P_{l}^{m}\left(\mu_{1}\right) P_{k}^{m}\left(\mu_{0}\right)(-1)^{l}\left\{Q_{l, k}^{m}\left(\mu_{1}\right)-(-1)^{l+k} Q_{l, k}^{m}\left(-\mu_{1}\right)\right\} \\
= & 0 \frac{(-\tau)^{2}}{2 !}+\cdots
\end{aligned}
$$

From the first and second terms in equation (27), the first power in $(-\tau)$ vanishes and the second power emerges only for the case $(l+k=e v e n)$.

From the third terms in the bracket of equation (27), we only need the first power in $(-\tau)$, because it is multiplied by another first power in $(-\tau)$. Substituting the equation (34) into this term, we obtain 


$$
\begin{aligned}
Q_{l, k}^{m}\left(-\mu_{0}\right)+(-1)^{l+k} Q_{l, k}^{m}\left(-\mu_{1}\right) & =2 q_{1,0}^{m l k}(-\tau)+\cdots, \quad(l+k=\text { even }) \\
& =0(-\tau)+\cdots \quad(l+k=\text { odd }) .
\end{aligned}
$$

From the fourth and fifth term, substituting the equation (34) we obtain following,

$$
\begin{aligned}
& \frac{1}{2} \sum_{a, b, c=m}^{\infty}(-1)^{a+b+c} w_{a}^{m} w_{b}^{m} w_{c}^{m} P_{a}^{m}\left(\mu_{1}\right) P_{c}^{m}\left(\mu_{0}\right) Q_{a, b}^{m}\left(\mu_{1}\right) Q_{b, c}^{m}\left(\mu_{0}\right) \\
- & \frac{1}{2} \sum_{a, b, c=m}^{\infty}(-1)^{b} w_{a}^{m} w_{b}^{m} w_{c}^{m} P_{a}^{m}\left(\mu_{1}\right) P_{c}^{m}\left(\mu_{0}\right) Q_{a, b}^{m}\left(-\mu_{1}\right) Q_{b, c}^{m}\left(-\mu_{0}\right) \\
= & 0(-\tau)^{2}+\cdots \quad(a+c=\text { even }) \\
= & {\left[\sum_{a, b, c=m}^{\infty}(-1)^{b+1} w_{a}^{m} w_{b}^{m} w_{c}^{m} P_{a}^{m}\left(\mu_{1}\right) P_{c}^{m}\left(\mu_{0}\right) q_{1,0}^{m a b} q_{1,0}^{m b c}\right](-\tau)^{2}+\cdots } \\
& (a+c=\text { odd })
\end{aligned}
$$

From the sixth term in the bracket of equation $(27)$, no $(-\tau)^{2}$ term emerges. Thus we obtain the truncated second iterations,

$$
\begin{aligned}
& \Delta S_{2}\left(\tau, \vec{i}_{1}, \vec{i}_{0}\right) \\
= & \sum_{m=0}^{\infty}\left(2-\delta_{0, m}\right)\left[\sum_{(l, k)^{1}}^{\infty}(-1)^{k} w_{l}^{m} w_{k}^{m}\left(q_{2,1}^{m l k}-2 q_{1,0}^{m l k}\right) P_{l}^{m}\left(\mu_{1}\right) P_{k}^{m}\left(\mu_{0}\right)\right. \\
+ & \left.2\left(\frac{1}{\mu_{1}}+\frac{1}{\mu_{0}^{-}}\right)^{-1} \sum_{(a, b, c)^{2}}^{\infty}(-1)^{b+1} w_{a}^{m} w_{b}^{m} w_{c}^{m} q_{1,0}^{m a b} q_{1,0}^{m b c} P_{a}^{m}\left(\mu_{1}\right) P_{c}^{m}\left(\mu_{0}\right)\right] \\
\times & \cos m\left(\varphi_{1}-\varphi_{0}\right) \frac{(-\tau)^{2}}{2 !}+\cdots,
\end{aligned}
$$

where $(l, k)^{1}$ denotes $l, k \geq m$ and $l+k$ is even and $(a, b, c)^{2}$ denotes $a, b, c \geq m$, $a+c$ is odd.

It is noted that the truncated second iteration does not have a first power in $(-\tau)$. It is also noted that the forth and fifth terms vanish for $w_{a}=0$ and $w_{a}=0$ to all the possible combination of $a+c=0$ such as Rayleigh scattering.

\subsection{Separated Integration}

In this subsection we consider changing order of lim and $\sum$ in the integration of the $\Delta S_{2}$. We begin with the integration of $U_{2}^{n}\left(\tau, i_{7}\right)$, 


$$
\begin{aligned}
& U_{2}^{n}\left(\tau, i_{7}\right)=\int_{0}^{1}\left(1-\exp \left(-\frac{\tau}{\mu}-\frac{\tau}{\mu_{7}}\right)\right) \frac{\mu_{7} \mu^{n} d \mu}{\mu_{7}+\mu} \\
= & -\lim _{\varepsilon \rightarrow 0} \int_{\varepsilon}^{1} \sum_{m=1}^{\infty} \frac{(-\tau)^{m}}{m !}\left(\frac{1}{\mu_{7}}+\frac{1}{\mu}\right)^{m-1} \mu^{n} \frac{d \mu}{\mu} .
\end{aligned}
$$

On the integral interval $(\varepsilon, 1)$, we interchange the order of $\int_{\varepsilon}^{1}$ and $\sum$ and divide the summation into two parts: one is $m \leq n$ and the other is $m>n$

$$
\begin{gathered}
U_{2}^{n}\left(\tau, i_{7}\right)=-\sum_{m=1}^{n}\left[\int_{0}^{1}\left(\frac{\mu}{\mu_{7}}+1\right)^{m-1} \mu^{n-m} d \mu\right] \frac{(-\tau)^{m}}{m !} \\
-(-\tau)^{n} \lim _{\varepsilon \rightarrow 0} \sum_{m=1}^{\infty} \int_{\varepsilon}^{1} \frac{(-\tau)^{m}}{(m+n) !}\left(\frac{1}{\mu_{7}}+\frac{1}{\mu}\right)^{m-1}\left(\frac{\mu}{\mu_{7}}+1\right)^{n} \frac{d \mu}{\mu} .
\end{gathered}
$$

The terms of the first part of the summation $(m \leq n)$ are ordinary integrations because the minimum powers in the $\mu$ in the integrand are equal to or greater than 0 and have no singularity at $\mu=0$. We can interchange the order of "lim" and " $\sum$ " for these terms. If the order is possible to exchange, we can "pick-up" each power in $(-\tau)$ separately. In other words, we can integrate the powers in $(-\tau)$ separately. We call this part as a Separated Integration Part (SIP).

The terms of the second part $(m>n)$ involve negative powers in $\mu$ or $\log p$, and have singularities at $\mu=0$. So we can not interchange the order of "lim" and " $\sum$ " and therefore we can not integrate the terms separately. We call the second part as latter a Non-Separated Integration Part (NIP). It is needless to say that the NIP converges as $\mu$ approaches to 0 , because it is equal to the converged $U_{2}^{n}\left(\tau, i_{6}\right)$ minus the bounded SIP.

We apply the above observation to equation (27).

\subsubsection{The First and Second Terms}

Due to the discussion in the previous subsection, we can select only the case $(l+k=$ even $)$ and pick up the second power in $(-\tau)^{2}$ only. $P_{l}^{m}\left(\mu_{3}\right) P_{k}^{m}\left(\mu_{3}\right)$ is a polynomial of $\mu_{3}$ and its lower powers for $l+k=$ even are given below (refer to appendix 2),

$$
\begin{aligned}
& P_{l}^{m}\left(\mu_{3}\right) P_{k}^{m}\left(\mu_{3}\right)=a_{0}+a_{2} \mu_{3}^{2}+\cdots, \quad(m+l=\text { even }), \\
& =a_{2} \mu_{3}^{2} \quad+a_{4} \mu_{3}^{4}+\cdots(m+l=o d d) .
\end{aligned}
$$


The zeroth power of $(-\tau), a_{0}$, is a NIP and the other higher powers of $(-\tau)$ are SIT. Taking account of $a_{0}=P_{l}^{m}(0) P_{k}^{m}(0)$, the integration above is then divided into SIP and NIP as below,

$$
\begin{gathered}
Q_{l, k}^{m}\left(\tau, \mu_{0}^{-}\right)=\lim _{\varepsilon \rightarrow 0} P_{l}^{m}(0) P_{k}^{m}(0) \int_{\varepsilon}^{1} U_{1}\left(\tau, \mu_{3}, \mu_{0}^{-}\right) \frac{d \mu_{3}}{\mu_{3}} \\
+\int_{0}^{1}\left(P_{l}^{m}\left(\mu_{3}\right) P_{k}^{m}\left(\mu_{3}\right)-P_{l}^{m}(0) P_{k}^{m}(0)\right) U_{1}\left(\tau, \mu_{3}, \mu_{0}^{-}\right) \frac{d \mu_{3}}{\mu_{3}} .
\end{gathered}
$$

Substituting the second power of $(-\tau)$ in the polynomial $U_{2}^{0}\left(p_{0}\right)$ into NIP and truncating SIP into the second power of $(-\tau)$, we obtain

$$
\begin{gathered}
Q_{l, k}^{m}\left(\tau, \mu_{0}^{-}\right)=\left[\left\{1+\left(C-\frac{1}{2}\right) \frac{1}{\mu_{0}^{-}}\right\} P_{l}^{m}(0) P_{k}^{m}(0)\right. \\
\left.-\int_{0}^{1}\left(P_{l}^{m}\left(\mu_{3}\right) P_{k}^{m}\left(\mu_{3}\right)-P_{l}^{m}(0) P_{k}^{m}(0)\right)\left(\frac{1}{\mu_{3}}+\frac{1}{\mu_{0}^{-}}\right) \frac{d \mu_{3}}{\mu_{3}}\right] \frac{(-\tau)^{2}}{2 !}+\cdots
\end{gathered}
$$

For the case $(m+l=o d d)$ the equation (45) holds becuse $P_{l}^{m}(0) P_{k}^{m}(0)=0$.

\subsubsection{The Third Term}

We select for the case $(l+k=e v e n)$ and pick up the first power of $(-\tau)$ only. We divide the integration into NIP and SIP, in the same as in equation (44). Substituting the first power of $(-\tau)$ in the polynomial $U_{2}^{0}\left(p_{0}\right)$ into NIP and truncating SIP into the first power of $(-\tau)$, we obtain

$$
\begin{gathered}
Q_{l, k}^{m}\left(\tau,-\mu_{0}^{-}\right)=\left[(C-1) P_{l}^{m}(0) P_{k}^{m}(0)\right. \\
\left.-\int_{0}^{1}\left(P_{l}^{m}\left(\mu_{3}\right) P_{k}^{m}\left(\mu_{3}\right)-P_{l}^{m}(0) P_{k}^{m}(0)\right) \frac{d \mu_{3}}{\mu_{3}}\right](-\tau)+\cdots+\cdots
\end{gathered}
$$

For the case $(m+l=o d d)$ the equation (46) holds becuse $P_{l}^{m}(0) P_{k}^{m}(0)=0$.

\subsubsection{The Fourth and Fifth Terms}

We integrate following for the case $(a+c=o d d)$, 


$$
\int_{0}^{1} \int_{0}^{1} P_{a}^{m}\left(\mu_{2}^{-}\right) P_{b}^{m}\left(\mu_{2}^{-}\right) P_{b}^{m}\left(\mu_{3}\right) P_{c}^{m}\left(\mu_{3}\right) U_{1}\left(\mu_{1}, \mu_{2}^{-}\right) \frac{d \mu_{2}^{-}}{\mu_{2}^{-}} U_{1}\left(\mu_{3}, \mu_{0}^{-}\right) \frac{d \mu_{3}}{\mu_{3}} .
$$

The lowest powers of $\mu_{2}^{-}$and $\mu_{3}$ for the product $P_{a}^{m}\left(\mu_{2}^{-}\right) \cdots$ are given below (refer to the appendix 2),

$$
\begin{array}{rlrl}
P_{a}^{m}\left(\mu_{2}^{-}\right) P_{b}^{m}\left(\mu_{2}^{-}\right) P_{b}^{m}\left(\mu_{3}\right) P_{c}^{m}\left(\mu_{3}\right) & \\
& =c_{12}\left(\mu_{2}^{-}\right)\left(\mu_{3}\right)^{2} & & (m+b=\text { odd }, a+b=\text { even }), \\
& =c_{21}\left(\mu_{2}^{-}\right)^{2}\left(\mu_{3}\right) & & (m+b=\text { odd }, a+b=\text { odd }), \\
& =c_{01}\left(\mu_{3}\right) & & (m+b=\text { even }, a+b=\text { even }), \\
& =c_{01}\left(\mu_{2}^{-}\right) & & (m+b=\text { even }, a+b=\text { odd }) .
\end{array}
$$

For $(m+b=o d d)$ the integration is the product of two SIP: one is for $\mu_{2}^{-}$and the other is for $\mu_{3}$. For $(m+b=$ even $)$ the integration is a product of one SIP and NIP. Then the integration is expressed for the four cases in the equation above:

$$
\begin{aligned}
= & {\left[\int_{0}^{1} \int_{0}^{1} P_{a}^{m}\left(\mu_{2}^{-}\right) P_{b}^{m}\left(\mu_{2}^{-}\right) P_{b}^{m}\left(\mu_{3}\right) P_{c}^{m}\left(\mu_{3}\right) \frac{d \mu_{2}^{-}}{\mu_{2}^{-}} \frac{d \mu_{3}}{\mu_{3}}\right](-\tau)^{2} } \\
+ & \cdot(m+b=\text { odd }), \\
= & {\left[-(C-1) P_{a}^{m}(0) P_{b}^{m}(0) \int_{0}^{1} P_{b}^{m}\left(\mu_{3}\right) P_{c}^{m}\left(\mu_{3}\right) \frac{d \mu_{3}}{\mu_{3}}\right.} \\
+ & \left.\int_{0}^{1} \int_{0}^{1}\left\{P_{a}^{m}\left(\mu_{2}^{-}\right) P_{b}^{m}\left(\mu_{2}^{-}\right)-P_{a}^{m}(0) P_{b}^{m}(0)\right\} P_{b}^{m}\left(\mu_{3}\right) P_{c}^{m}\left(\mu_{3}\right) \frac{d \mu_{2}^{-}}{\mu_{2}^{-}} \frac{d \mu_{3}}{\mu_{3}}\right](-\tau)^{2} \\
& +\cdots \\
= & {\left[-(C-1) P_{b}^{m}(0) P_{c}^{m}(0) \int_{0}^{1} P_{a}^{m}\left(\mu_{2}^{-}\right) P_{b}^{m}\left(\mu_{2}^{-}\right) \frac{d \mu_{2}^{-}}{\mu_{2}^{-}}\right.} \\
+ & \left.\int_{0}^{1} \int_{0}^{1} P_{a}^{m}\left(\mu_{2}^{-}\right) P_{b}^{m}\left(\mu_{2}^{-}\right)\left\{P_{b}^{m}\left(\mu_{3}\right) P_{c}^{m}\left(\mu_{3}\right)-P_{b}^{m}(0) P_{c}^{m}(0)\right\} \frac{d \mu_{2}^{-}}{\mu_{2}^{-}} \frac{d \mu_{3}}{\mu_{3}}\right](-\tau)^{2} \\
& +\cdots \\
& \cdots
\end{aligned}
$$

\subsection{Surface Integration}

We can bring back the idea of division of integration to the surface integration on the unit sphere expressed in equation (19). This division is possible, 
because the integrations in equation (19) are expressed as the summation of the integrations of the products of Bi-Legendre functions shown in equation (27). From the nineth integration in equation (19) no second power in $(-\tau)^{2}$ emerges.

\subsubsection{The first four integrations}

The first integration is divided into SIP and NIP expressed as below,

$$
\begin{aligned}
& \int P\left(\vec{i}_{1}, \vec{i}_{3}\right) P\left(\vec{i}_{3}, \vec{i}_{0}\right) U_{1}\left(\tau, \mu_{0}^{-}, \mu_{3}\right) \frac{d \Omega_{3}}{4 \pi \mu_{3}} \\
= & \int\left\{P\left(\overrightarrow{i_{1}}, \overrightarrow{i_{3}}\right) P\left(\vec{i}_{3}, \vec{i}_{0}\right)-P\left(\vec{i}_{1}, \vec{i}_{3}^{\prime}\right) P\left(\vec{i}_{3}^{\prime}, \vec{i}_{0}\right)\right\} U_{1}\left(\tau, \mu_{0}^{-}, \mu_{3}\right) \frac{d \Omega_{3}}{4 \pi \mu_{3}} \\
+ & \lim _{\mu_{3} \rightarrow 0} \int\left\{P\left(\vec{i}_{1}, \vec{i}_{3}^{\prime}\right) P\left(\vec{i}_{3}^{\prime}, \vec{i}_{0}\right)\right\} U_{1}\left(\tau, \mu_{0}^{-}, \mu_{3}\right) \frac{d \Omega_{3}}{4 \pi \mu_{3}} .
\end{aligned}
$$

where $\vec{i}_{3}^{\prime}$ denotes the projected direction of $\vec{i}_{3}$ onto the equator in the unit sphere. Substituting the second power of $(-\tau)$ in the polynomial $U_{2}^{0}\left(p_{0}\right)$ and the truncated term of the second power into equation (50), we can obtain

$$
\begin{aligned}
& \int P\left(\vec{i}_{1}, \vec{i}_{3}\right) P\left(\vec{i}_{3}, \vec{i}_{0}\right) U_{1}\left(\tau, \mu_{0}^{-}, \mu_{3}\right) \frac{d \Omega_{3}}{4 \pi \mu_{3}} \\
= & -\left[\int\left\{P\left(\vec{i}_{1}, \vec{i}_{3}\right) P\left(\vec{i}_{3}, \vec{i}_{0}\right)-P\left(\vec{i}_{1}, \vec{i}_{3}^{\prime}\right) P\left(\vec{i}_{3}^{\prime}, \vec{i}_{0}\right)\right\}\left(\frac{1}{\mu_{0}^{-}}+\frac{1}{\mu_{3}}\right) \frac{d \Omega_{3}}{4 \pi \mu_{3}}\right] \frac{(-\tau)^{2}}{2 !} \\
+ & \left\{1+\left(C-\frac{1}{2}\right) \frac{1}{\mu_{0}^{-}}\right\}\left[\int_{0}^{2 \pi} P\left(\vec{i}_{1}, \vec{i}_{3}^{\prime}\right) P\left(\vec{i}_{3}^{\prime}, \vec{i}_{0}\right) \frac{d \varphi_{3}}{4 \pi}\right] \frac{(-\tau)^{2}}{2 !} .
\end{aligned}
$$

Substituting the above equations into the first and second integrations in the bracket in equation (19), we obtain

$$
\begin{aligned}
& \int P\left(\vec{i}_{1}, \vec{i}_{3}\right) P\left(\vec{i}_{3}, \vec{i}_{0}\right) U_{1}\left(\mu_{0}^{-}\right) \frac{d \Omega_{3}}{4 \pi \mu_{3}}-\int P\left(\vec{i}_{1}, \vec{i}_{3}\right) P\left(\vec{i}_{3}, \vec{i}_{0}\right) U_{1}\left(-\mu_{1}\right) \frac{d \Omega_{3}}{4 \pi \mu_{3}} \\
= & -\left(\frac{1}{\mu_{0}^{-}}+\frac{1}{\mu_{1}}\right)\left[\int\left\{P\left(\vec{i}_{1}, \vec{i}_{3}\right) P\left(\vec{i}_{3}, \vec{i}_{0}\right)-P\left(\vec{i}_{1}, \vec{i}_{3}^{\prime}\right) P\left(\vec{i}_{3}^{\prime}, \vec{i}_{0}\right)\right\} \frac{d \Omega_{3}}{4 \pi \mu_{3}}\right] \frac{(-\tau)^{2}}{2 !} \\
+ & \left(C-\frac{1}{2}\right)\left(\frac{1}{\mu_{1}}+\frac{1}{\mu_{0}^{-}}\right)\left[\int_{0}^{2 \pi} P\left(\vec{i}_{1}, \vec{i}_{3}^{\prime}\right) P\left(\vec{i}_{3}^{\prime}, \vec{i}_{0}\right) \frac{d \varphi_{3}}{4 \pi}\right] \frac{(-\tau)^{2}}{2 !} .
\end{aligned}
$$

We evaluate the third and fourth integrations in equation (19) in the same 
manner.

\subsubsection{The fifth and sixth integrations}

The fifth integration is devided into SIP and NIP as below,

$$
\begin{aligned}
& -\left\{\exp \left(-\frac{\tau}{\mu_{0}^{-}}-\frac{\tau}{\mu_{1}}\right)-1\right\} \int P\left(\vec{i}_{1}, \vec{i}_{3}\right) P\left(\vec{i}_{3}, \vec{i}_{0}\right) U_{1}\left(-\mu_{1}\right) \frac{d \Omega_{3}}{4 \pi \mu_{3}} \\
= & -2\left(\frac{1}{\mu_{1}}+\frac{1}{\mu_{0}^{-}}\right)\left[(C-1) \int_{0}^{2 \pi} P\left(\vec{i}_{1}, \vec{i}_{3}^{\prime}\right) P\left(\vec{i}_{3}^{\prime}, \vec{i}_{0}\right) \frac{d \varphi_{3}}{4 \pi}\right. \\
- & \left.\int\left\{P\left(\vec{i}_{1}, \vec{i}_{3}\right) P\left(\vec{i}_{3}, \vec{i}_{0}\right)-P\left(\vec{i}_{1}, \vec{i}_{3}^{\prime}\right) P\left(\vec{i}_{3}^{\prime}, \vec{i}_{0}\right)\right\} \frac{d \Omega_{3}}{4 \pi \mu_{3}}\right] \frac{(-\tau)^{2}}{2 !}+\cdots
\end{aligned}
$$

The equation above holds for the two cases described in (46). We can evaluate the sixth integration in equation (19) in the same manner.

\subsubsection{The seventh and eighth integrations}

The seventh integration is devided into SIP and NIP as below,

$$
\begin{aligned}
& \iint P\left(\vec{i}_{1}, \vec{i}_{2}\right) P\left(\vec{i}_{2}, \vec{i}_{3}\right) P\left(\vec{i}_{3}, \vec{i}_{0}\right) U_{1}\left(\mu_{1}, \mu_{2}^{-}\right) U_{1}\left(\mu_{3}, \mu_{0}^{-}\right) \frac{d \Omega_{2}^{-}}{4 \pi \mu_{2}^{-}} \frac{d \Omega_{3}}{4 \pi \mu_{3}} \\
& =\iint\left[P\left(\vec{i}_{1}, \vec{i}_{2}\right) P\left(\vec{i}_{2}, \vec{i}_{3}\right) P\left(\vec{i}_{3}, \vec{i}_{0}\right)-P\left(\vec{i}_{1}, \vec{i}_{2}^{\prime}\right) P\left(\vec{i}_{2}^{\prime}, \vec{i}_{3}\right) P\left(\vec{i}_{3}, \vec{i}_{0}\right)\right. \\
& \left.-P\left(\vec{i}_{1}, \vec{i}_{2}\right) P\left(\vec{i}_{2}, \vec{i}_{3}^{\prime}\right) P\left(\vec{i}_{3}^{\prime}, \vec{i}_{0}\right)\right] U_{1}\left(\mu_{1}, \mu_{2}^{-}\right) U_{1}\left(\mu_{3}, \mu_{0}^{-}\right) \frac{d \Omega_{2}^{-}}{4 \pi \mu_{2}^{-}} \frac{d \Omega_{3}}{4 \pi \mu_{3}} \\
& +\iint P\left(\vec{i}_{1}, \vec{i}_{2}^{\prime}\right) P\left(\vec{i}_{2}^{\prime}, \vec{i}_{3}\right) P\left(\vec{i}_{3}, \vec{i}_{0}\right) U_{1}\left(\mu_{1}, \mu_{2}^{-}\right) U_{1}\left(\mu_{3}, \mu_{0}^{-}\right) \\
& +\iint P\left(\vec{i}_{1}, \overrightarrow{i_{2}}\right) P\left(\overrightarrow{i_{2}}, \vec{i}_{3}^{\prime}\right) P\left({\overrightarrow{i_{3}^{\prime}}}_{3}, \vec{i}_{0}\right) U_{1}\left(\mu_{1}, \mu_{2}^{-}\right) U_{1}\left(\left(\mu_{3}, \mu_{0}^{-}\right)\right] \frac{d \Omega_{2}^{-}}{4 \pi \mu_{2}^{-}} \frac{d \Omega_{3}}{4 \pi \mu_{3}} \\
& =\left[\int \int \left\{P\left(\vec{i}_{1}, \vec{i}_{2}\right) P\left(\vec{i}_{2}, \vec{i}_{3}\right) P\left(\vec{i}_{3}, \vec{i}_{0}\right)-P\left(\vec{i}_{1}, \vec{i}_{2}^{\prime}\right) P\left(\vec{i}_{2}^{\prime}, \vec{i}_{3}\right) P\left(\vec{i}_{3}, \vec{i}_{0}\right)\right.\right. \\
& \left.-P\left(\vec{i}_{1}, \vec{i}_{2}\right) P\left(\vec{i}_{2}, \vec{i}_{3}^{\prime}\right) P\left(\vec{i}_{3}^{\prime}, \vec{i}_{0}\right)\right\} \frac{d \Omega_{2}^{-}}{4 \pi \mu_{2}^{-}} \frac{d \Omega_{3}}{4 \pi \mu_{3}} \\
& -(C-1) \iint P\left(\overrightarrow{i_{1}}, \overrightarrow{i_{2}^{\prime}}\right) P\left(\overrightarrow{i_{2}^{\prime}}, \overrightarrow{i_{3}}\right) P\left(\vec{i}_{3}, \overrightarrow{i_{0}}\right) \frac{d \varphi_{2}}{4 \pi} \frac{d \Omega_{3}}{4 \pi \mu_{3}} \\
& \left.-(C-1) \iint P\left(\vec{i}_{1}, \vec{i}_{2}\right) P\left(\vec{i}_{2}, \vec{i}_{3}^{\prime}\right) P\left(\vec{i}_{3}^{\prime}, \vec{i}_{0}\right) \frac{d \Omega_{2}^{-}}{4 \pi \mu_{2}^{-}} \frac{d \varphi_{3}}{4 \pi}\right](-\tau)^{2} .
\end{aligned}
$$

The equation above holds for all the cases described in (49). We can evaluate 
the eighth integration in equation (19) in the same manner.

\subsection{The Result of the Second Approximation}

Expanding the first approximated scattering function into a power series expansion in $\tau$ and truncating up to the second degree, we obtain

$$
S_{1}\left(\tau, \vec{i}_{1}, \vec{i}_{0}\right)=\left[\tau-\left(\frac{1}{\mu_{1}}+\frac{1}{\left|\mu_{0}\right|}\right) \frac{\tau^{2}}{2 !}\right] P\left(\vec{i}_{1}, \vec{i}_{0}\right) .
$$

The second iteration is expressed by the integration of the products of the phase functions as below,

$$
\begin{gathered}
\Delta S_{2}\left(\tau, \vec{i}_{1}, \vec{i}_{0}\right)=\left[(3-2 C) I_{e}\left(\overrightarrow{i_{1}}, \vec{i}_{0}\right)+I_{u}\left(\vec{i}_{1}, \vec{i}_{0}\right)+I_{l}\left(\vec{i}_{1}, \vec{i}_{0}\right)\right. \\
\left.+2\left(\frac{1}{\mu_{1}}+\frac{1}{\mu_{0}^{-}}\right)^{-1}\left\{(C-1)\left(I_{u u}\left(\vec{i}_{1}, \vec{i}_{0}\right)+I_{l l}\left(\vec{i}_{1}, \vec{i}_{0}\right)\right)+I_{u l}^{2}\left(\vec{i}_{0}, \vec{i}_{1}\right)\right\}\right] \frac{(-\tau)^{2}}{2 !} .
\end{gathered}
$$

The coefficients are evaluated on the half unit sphere (we return the variable $\mu_{2}^{-}$to $\left.\mu_{2}\right)$,

$$
\begin{aligned}
& I_{u}\left(\vec{i}_{1}, \vec{i}_{0}\right)=\int_{U}\left\{P\left(\vec{i}_{1}, \vec{i}_{3}\right) P\left(\vec{i}_{3}, \vec{i}_{0}\right)-P\left(\vec{i}_{1}, \vec{i}_{3}^{\prime}\right) P\left(\vec{i}_{3}^{\prime}, \vec{i}_{0}\right)\right\} \frac{d \Omega_{3}}{4 \pi \mu_{3}}, \\
& I_{l}\left(\vec{i}_{1}, \vec{i}_{0}\right)=\int_{L}\left\{P\left(\vec{i}_{1}, \vec{i}_{2}\right) P\left(\vec{i}_{2}, \vec{i}_{0}\right)-P\left(\vec{i}_{1}, \vec{i}_{2}^{\prime}\right) P\left(\vec{i}_{2}^{\prime}, \vec{i}_{0}\right)\right\} \frac{d \Omega_{2}}{4 \pi\left|\mu_{2}\right|}, \\
& I_{e}\left(\vec{i}_{1}, \vec{i}_{0}\right)=\int_{0}^{2 \pi} P\left(\overrightarrow{i_{1}}, \vec{i}_{3}^{\prime}\right) P\left(\vec{i}_{3}^{\prime}, \vec{i}_{0}\right) \frac{d \varphi_{3}}{4 \pi}, \\
& I_{u u}\left(\vec{i}_{1}, \vec{i}_{0}\right)=\int_{U} \int_{0}^{2 \pi}\left\{P\left(\vec{i}_{1}, \vec{i}_{2}^{\prime}\right) P\left(\vec{i}_{2}^{\prime}, \vec{i}_{3}\right) P\left(\vec{i}_{3}, \vec{i}_{0}\right)-P\left(\vec{i}_{0}, \vec{i}_{2}^{\prime}\right) P\left(\vec{i}_{2}^{\prime}, \vec{i}_{3}\right) P\left(\vec{i}_{3}, \vec{i}_{1}\right)\right\} \frac{d \varphi_{2} d \Omega_{3}}{16 \pi^{2} \mu_{3}}, \\
& I_{l l}\left(\overrightarrow{i_{1}}, \overrightarrow{i_{0}}\right)=\int_{L} \int_{0}^{2 \pi}\left\{P\left(\overrightarrow{i_{1}}, \overrightarrow{i_{2}}\right) P\left(\overrightarrow{i_{2}}, \overrightarrow{i_{3}^{\prime}}\right) P\left(\overrightarrow{i_{3}^{\prime}}, \overrightarrow{i_{0}}\right)-P\left(\overrightarrow{i_{0}}, \overrightarrow{i_{2}}\right) P\left(\overrightarrow{i_{2}}, \overrightarrow{i_{3}^{\prime}}\right) P\left(\overrightarrow{i_{3}^{\prime}}, \overrightarrow{i_{1}}\right)\right\} \frac{d \varphi_{3} d \Omega_{2}}{16 \pi^{2}\left|\mu_{2}\right|}, \\
& I_{u l}\left(\vec{i}_{1}, \vec{i}_{0}\right)=\int_{U} \int_{L}\left[\left\{P\left(\vec{i}_{1}, \vec{i}_{2}\right) P\left(\vec{i}_{2}, \vec{i}_{3}\right) P\left(\vec{i}_{3}, \vec{i}_{0}\right)-P\left(\vec{i}_{0}, \vec{i}_{2}\right) P\left(\vec{i}_{2}, \vec{i}_{3}\right) P\left(\vec{i}_{3}, \vec{i}_{1}\right)\right\},\right. \\
& -\left\{P\left(\vec{i}_{1}, \vec{i}_{2}^{\prime}\right) P\left(\vec{i}_{2}^{\prime}, \vec{i}_{3}\right) P\left(\vec{i}_{3}, \vec{i}_{0}\right)-P\left(\vec{i}_{0}, \vec{i}_{2}^{\prime}\right) P\left(\vec{i}_{2}^{\prime}, \vec{i}_{3}\right) P\left(\vec{i}_{3}, \vec{i}_{1}\right)\right\}
\end{aligned}
$$




$$
\left.-\left\{P\left(\vec{i}_{1}, \vec{i}_{2}\right) P\left(\vec{i}_{2}, \vec{i}_{3}^{\prime}\right) P\left(\vec{i}_{3}^{\prime}, \vec{i}_{0}\right)-P\left(\vec{i}_{0}, \vec{i}_{2}\right) P\left(\vec{i}_{2}, \vec{i}_{3}^{\prime}\right) P\left(\vec{i}_{3}^{\prime}, \vec{i}_{1}\right)\right\}\right] \frac{d \Omega_{2} d \Omega_{3}}{16 \pi^{2}\left|\mu_{2}\right| \mu_{3}} .
$$

Adding the first approximation to the second iteration, we obtain the second approximation,

$$
\begin{aligned}
& S_{2}\left(\tau, \vec{i}_{1}, \vec{i}_{0}\right)=P\left(\vec{i}_{1}, \vec{i}_{0}\right) \tau-\left(\frac{1}{\mu_{1}}+\frac{1}{\left|\mu_{0}\right|}\right) P\left(\vec{i}_{1}, \vec{i}_{0}\right) \frac{(-\tau)^{2}}{2 !} \\
+ & \left\{-2 I_{e}\left(\vec{i}_{1}, \vec{i}_{0}\right)+2\left(\frac{1}{\mu_{1}}+\frac{1}{\left|\mu_{0}\right|}\right)^{-1}\left(I_{u u}\left(\vec{i}_{1}, \vec{i}_{0}\right)+I_{l l}\left(\vec{i}_{1}, \vec{i}_{0}\right)\right)\right\} \log \tau \frac{(-\tau)^{2}}{2 !} \\
+ & {\left[I_{u}\left(\vec{i}_{1}, \vec{i}_{0}\right)+I_{l}\left(\vec{i}_{1}, \vec{i}_{0}\right)+(3-2 \gamma) I_{e}\left(\vec{i}_{1}, \vec{i}_{0}\right)\right.} \\
+ & \left.2\left(\frac{1}{\mu_{1}}+\frac{1}{\left|\mu_{0}\right|}\right)^{-1}\left\{(\gamma-1)\left(I_{u u}\left(\vec{i}_{1}, \vec{i}_{0}\right)+I_{l l}\left(\vec{i}_{1}, \vec{i}_{0}\right)\right)+I_{u l}\left(\vec{i}_{1}, \vec{i}_{0}\right)\right\}\right] \frac{(-\tau)^{2}}{2 !} .
\end{aligned}
$$

From the above equation, we summarize the characteristics of the second approximation.

(1)The second approximation is not a quadratic equation of the optical thickness $\tau$ but a quadratic equation with a $\tau^{2} \log (\tau)$.

(2)The second iteration $\Delta S_{2}\left(\tau, \vec{i}_{1}, \vec{i}_{0}\right)$ does not have the first power of $\tau$.

(3)There exist three kinds of terms which are characterized by the number of multiplication of the scattering phase function: single scattering, double scattering and triple scattering. The single scattering term comes from the first approximation. The surface integral $I_{e}, I_{l}, I_{u}$ are the double scattering terms and $I_{u u}, I_{l l}, I_{u l}$ are the triple scattering terms.

(4) For the atmosphere with a scattering phase function which does not have the odd order Legendre functions in its series expansion in the cosine of the angle, such as the Rayleigh scattering, there are no triple scattering terms in the second approximation.

\section{Calculation of Scattering}

To evaluate the accuracy of the second approximation discussed in the previous sections, we carry out calculations for the Rayleigh and aerosol scattering atmospheres for various cases and compare with the exact solutions. To appreciate the improvement of accuracy from single scattering, we calculate the 


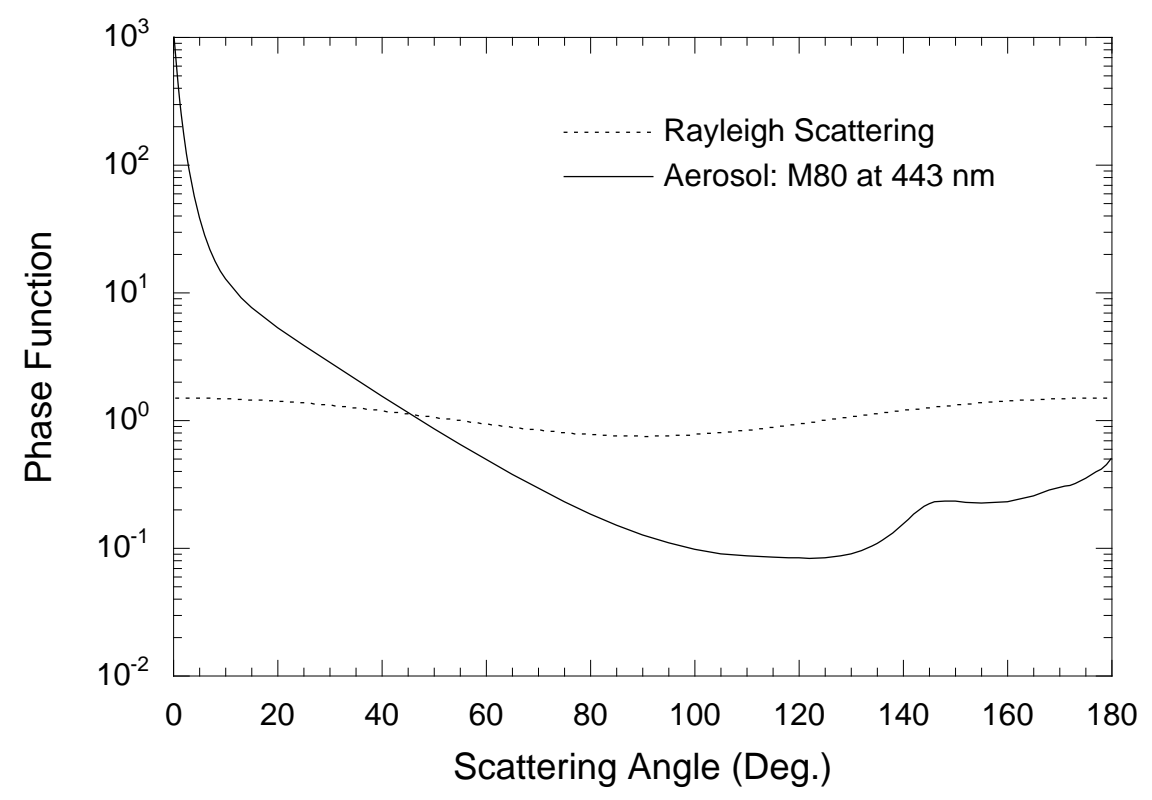

Fig. 2. Phase Functions

the single scattering reflectance as,

$$
\rho\left(\lambda, \vec{i}_{1}, \vec{i}_{0}\right)=\frac{\tau(\lambda) \omega(\lambda) P\left(i_{1}, i_{0}\right)}{4 \mu_{1}\left|\mu_{0}\right|},
$$

where $\lambda$ is the wavelength and $\omega(\lambda)$ is the single scattering albedo. Figure 2 illustrates the phase function of air molecules (Raleigh scattering) as well as for a typical maritime aerosol with relative humidity $(\mathrm{RH})$ of $80.0 \%$ (denoted as M80) at wavelength $443 \mathrm{~nm}$. The Maritime aerosol model has a single scattering albedo of 0.9929 , and the Rayleigh scattering model has a single scattering albedo of 1.0. Note that, in contrast to Rayleigh scattering function which is symmetry in the forward and backward smatterings, the maritime aerosol predominantly scatters in the forward direction.

\subsection{The Exact Solutions}

The successive-order-of-scattering (SOS) [4] method is used in solving the radiative transfer equation (RTE) for the Rayleigh scattering atmosphere and the aerosol scattering atmosphere. The SOS code for the ocean-atmosphere system was developed for the atmospheric correction algorithm for the ocean color sensors[5,6]. This code computes the upward radiance at the top and the downward radiance at the base of the medium for the ocean-atmosphere system. The code is capable of yielding radiances that are accurate to nearly $0.1 \%$ [5]. In the following two subsections, the reflectance computed by the single scattering and the second approximation are compared with the exact 

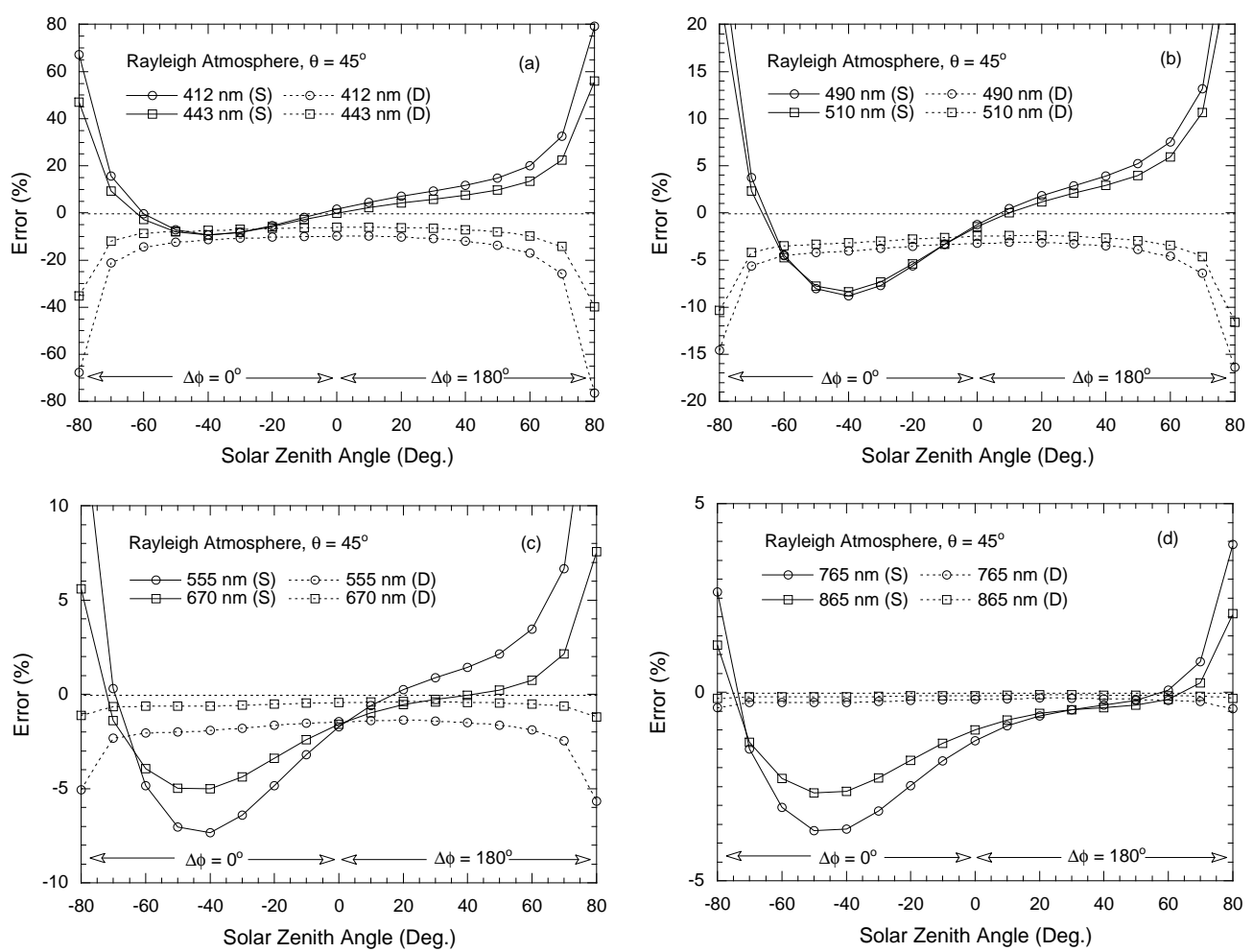

Fig. 3. Comparison for Rayleigh Scattering

solutions obtained using the SOS code.

\subsection{The Rayleigh Scattering Atmosphere}

Figure 3 provides a quantitative evaluation of accuracy using the second approximation for a Rayleigh scattering atmosphere for the various solar-sensor geometries and for the eight wavelengths at 412, 443, 490, 510, 670, 765, and $865 \mathrm{~nm}$. The Rayleigh optical thicknesses corresponding to the eight wavelengths from 412 to $865 \mathrm{~nm}$ are $0.3185,0.2361,0.1560,0.1324,0.0938,0.0436$, 0.0255 and 0.0155 respectively. In all computations, the Rayleigh atmosphere is treated as a plane-parallel medium without a surface boundary. Figures 3(a)-(d) show the error (\%) in the computed upward reflectance as a function of the solar zenith angle and for a sensor viewing angle of $45^{\circ}$. The left and right parts of each plot in Figure 3 corresponds to the relative azimuth angles of $0^{\circ}$ and $180^{\circ}$ (principal scattering plane), respectively.

Results in Figure 3 show the significant improvement in accuracy using the second approximation compared with the single scattering formula. Using the new formula, the errors are all within $1 \%$ for the red and near-infrared wavelengths, while errors are within nearly $10 \%$ for the blue bands for zenith angles less than $60^{\circ}$. It is important to note that, with the second approximation, 

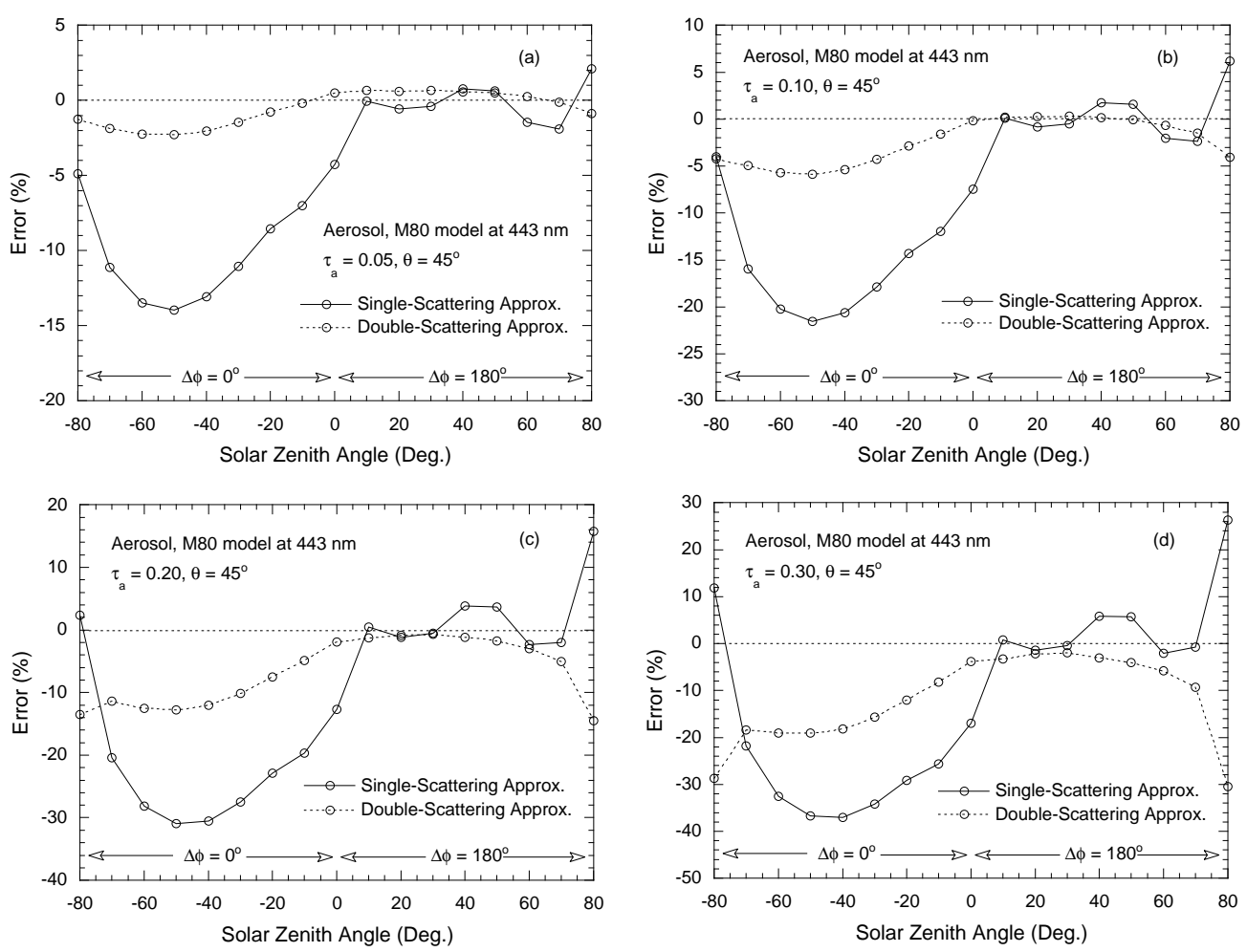

Fig. 4. Comparison of Calculation the Maritime Aerosol

the error in a given wavelength(optical thickness) is almost independent of the solar and sensor viewing geometry.

\subsection{The Maritime Aerosol Atmosphere}

A realistic maritime aerosol model as in Figure 2 is used to study the efficacy of the double scattering approximation in computing the upward reflectance. The double scattering approximation is the second approximation without triple scattering. Computations for various coefficients are much more involved due predominantly to the aerosol forward scattering characteristics. Figure 4 shows examples of error (\%) for sensor viewing angle of $45^{\circ}$ as a function of the solar zenith angle for the principal scattering plane. Figure 4(a)-(d) are the results for the aerosol optical thickness of $0.05,0.1,0.2$, and 0.3 respectively. Again, we includ results from the single scattering approximation given by equation (64) for comparison. Similar to the Rayleigh atmosphere case, the aerosol atmosphere is considered as one plane-parallel layer without the surface boundary.

The results in Figure 4 show significant improvement in accuracy using the double scattering approximation compared with the single scattering formula, in particular, for the part of the results with $\Delta \phi=0^{\circ}$. It is interesting to 
note that the scattering angles for the $\Delta \phi=0^{\circ}$ part ( the left part of the plot) vary from $55^{\circ}-135^{\circ}$. (all forward scattering), while the scattering angles for $\Delta \phi=180^{\circ}$ (the right part of the plot) change from $135^{\circ}-180^{\circ}$ (all backward scattering). We are usually more interested in the results with the large scattering angles because measurements with the large scattering angles are generally accessible for satellite remote sensing. With double scattering approximation and scattering angles $>100^{\circ}$, the reflectance error is usually within about $1 \%$ for the aerosol optical thickness of 0.05 , while the error is within about $4 \%$ for the optical thickness of 0.1 . As expected, for the turbid atmosphere, the error increases. The error is proportional to the slant path of the optical thickness, i.e., $\tau_{a} / \cos \theta$. The aerosol reflectance errors are within about $7 \%$ and about $12 \%$ for the optical thickness of 0.2 and 0.3 respectively.

\section{Conclusion}

We obtain a new approximated solution in the radiative transfer problem in the anisotropic plane-parallel atmosphere by iterating integration of Chandrasekhar's integral equation.

By the second approximation, the scattering function $S\left(\tau, \overrightarrow{i_{1}}, \overrightarrow{i_{0}}\right)$ is expressed as a quadratic equation in the optical thickness, $\tau$, with terms $\tau^{2} \log (\tau)$. It is noted that the linear term in $\tau$ in the second approximation is identical to the first approximation. This implies that the iterative integration method improves the accuracy from the first approximation and approachs to the solution of radiative transfer.

The second approximation is not a pure quadratic equation but a quadratic equation with terms $\tau^{2} \log (\tau)$. The "log" term comes from the singularity at the lower integral limit. It is possible that, by expanding $\log \tau$ around $\tau \neq 0.0$, we can obtain the quadratic equation form of the scattering function $S\left(\tau, \vec{i}_{1}, \vec{i}_{0}\right)$. Rather we leave it as "log" to insist the validity of the expression in the range $\tau \geq 0.0$.

The coefficients of $\tau^{2}$ and $\tau^{2} \log (\tau)$ are evaluated by surface integrations of products of the phase functions on the half unit sphere.

The generalized upward reflectances numerically evaluated by the second approximation for the Rayleigh atmosphere and the marinetime aerosol atmosphere are very much closer to the exact solution than the single scattering approximation. In some cases, such as the small optical thickness, they are sufficiently close to the exact solution. 
Acknowledgment This work is done during Tanaka's stay at NASA Goddard Space Flight Center as a visiting scientist. The authors expresses their sincere appreciation to the SIMBIOS project people for their assistances.

\section{References}

[1] J. E. Hansen and L. D. Travis, Light scattering in planetary atmosphere, Space Science Review16 (1974) 527-610

[2] R. M. Goody and Y. L. Yung, Atmospheric Radiation, Oxford University Press, New York Oxford, (1989)

[3] S. Chandrasekhar, "RADIATIVE TRANSFER", Dover Publishing, Inc., (1960).

[4] H.C.van de Hulst, "Multiple Light Scattering", Academic Press, New York, (1980)

[5] H. R. Gordon, M. Wang "Surface roughness considerations for atmospheric correction of ocean color sensors. I: The Rayleigh scattering component", Appl. Opt., vol. 31, pp.4247-4260, (1992)

[6] H. R. Gordon, M. Wang "Retrieval of water-leaving radiance and aerosol optical thickness over the oceans with SeaWiFS preliminary algorithm", Appl. Opt., vol. 33, pp.443-452, (1994)

[7] T. Tanaka "Integration of Chandrasekhar's Integral Equation", Journal of Quantitative Spectroscopy and Radiative Transfer(in press), (2002)

\section{Appendix 1, Derivation of Function $U_{2}^{n}(\tau, \mu)$}

We define a new function $W_{n}(\tau)$ as

$$
W_{n}(\tau)=\int_{0}^{1} \mu_{3}^{n} \exp \left(-\frac{\tau}{\mu_{3}}\right) d \mu_{3}
$$

$W_{0}(\tau)$ is evaluated 


$$
\begin{aligned}
& W_{0}(\tau)=\int_{0}^{1} \exp \left(-\frac{\tau}{\mu_{3}}\right) d \mu_{3}=\left[\mu_{3} \exp \left(-\frac{\tau}{\mu_{3}}\right)\right]_{0}^{1}-\int_{0}^{1} \mu_{3}\left\{\exp \left(-\frac{\tau}{\mu_{3}}\right)\right\}^{\prime} d \mu_{3} \\
= & \exp (-\tau)-(-\tau)(C+\operatorname{eax}(\tau)) .
\end{aligned}
$$

The recurrence relation of $W_{n}(\tau)$ is given as

$$
W_{n}(\tau)=\frac{\exp (-\tau)}{n+1}+\frac{(-\tau)}{n+1} W_{n-1}(\tau)
$$

We can evaluate $W_{n}(\tau)$ by the recurrence relation and the initial function $W_{0}(\tau)$. Using $W_{n}(\tau)$, we can derive the recurrence relation for $U_{2}^{n}(\tau, \mu)$

$$
U_{2}^{n}\left(\tau, \mu_{0}\right)=\mu_{0}\left[\frac{1}{n}-\exp \left(-\frac{\tau}{\mu_{0}}\right) W_{n-1}-U_{2}^{n-1}\left(\mu_{0}\right)\right]
$$

Truncating the series expansion up to the second degree in $(-\tau)$, we obtain

$$
\begin{aligned}
& U_{2}^{1}\left(\tau, \mu_{0}\right)=-(-\tau)-\frac{3(-\tau)^{2}}{4}+C \frac{(-\tau)^{2}}{2}-\frac{1}{2} \frac{(-\tau)^{2}}{\mu_{0}} \\
& U_{2}^{n}\left(\tau, \mu_{0}\right)=-\frac{(-\tau)}{n}-\frac{(-\tau)^{2}}{2(n-1)}-\frac{1}{2 n} \frac{(-\tau)^{2}}{\mu_{0}} . \quad(n \geq 2)
\end{aligned}
$$

\section{Appendix 2, Lower powers of the product of the Bi-Legendre func- tion}

$$
\begin{aligned}
& m=e, \quad l=e, \quad k=e, \quad P_{l}^{m}\left(\mu_{3}\right) P_{k}^{m}\left(\mu_{3}\right)=a_{0} \quad+a_{2} \mu_{3}^{2} \\
& m=e, \quad l=o, \quad k=o, \quad P_{l}^{m}\left(\mu_{3}\right) P_{k}^{m}\left(\mu_{3}\right)=\quad a_{2} \mu_{3}^{2} \quad+a_{4} \mu_{3}^{4} \\
& m=e, \quad l=e, \quad k=o, \quad P_{l}^{m}\left(\mu_{3}\right) P_{k}^{m}\left(\mu_{3}\right)=a_{1} \mu_{3} \quad+a_{3} \mu_{3}^{3} \\
& m=e, \quad l=o, \quad k=e, \quad P_{l}^{m}\left(\mu_{3}\right) P_{k}^{m}\left(\mu_{3}\right)=a_{1} \mu_{3} \quad+a_{3} \mu_{3}^{3} \\
& m=o, \quad l=e, \quad k=e, \quad P_{l}^{m}\left(\mu_{3}\right) P_{k}^{m}\left(\mu_{3}\right)=\quad a_{2} \mu_{3}^{2} \quad+a_{4} \mu_{3}^{4} \\
& m=o, \quad l=o, \quad k=o, \quad P_{l}^{m}\left(\mu_{3}\right) P_{k}^{m}\left(\mu_{3}\right)=a_{0} \quad+a_{2} \mu_{3}^{2} \\
& m=o, \quad l=e, \quad k=o, \quad P_{l}^{m}\left(\mu_{3}\right) P_{k}^{m}\left(\mu_{3}\right)=a_{1} \mu_{3} \quad+a_{3} \mu_{3}^{3} \\
& m=o, \quad l=o, \quad k=e, \quad P_{l}^{m}\left(\mu_{3}\right) P_{k}^{m}\left(\mu_{3}\right)=a_{1} \mu_{3} \quad+a_{3} \mu_{3}^{3}
\end{aligned}
$$

\section{Figute Captions}


Figure 1: Geometry of Radiative Transfer

Figure 2: Scattering Phase Functions

Figure 3: Calculation of Generalized Reflectances for Rayleigh Scattering

Figure 4: Calculation of Generalized Reflectances for Maritime Aerosol Scattering 